This is a self-archived - parallel published version of this article in the publication archive of the University of Vaasa. It might differ from the original.

\title{
Cancer Modeling-on-a-Chip with Future Artificial Intelligence Integration
}

Author(s): Fetah, Kirsten Lee; DiPardo, Benjamin J.; Kongadzem, EveMary; Tomlinson, James S.; Elzagheid, Adam; Elmusrati, Mohammed; Khademhosseini, Ali; Ashammakhi, Nureddin

Title: Cancer Modeling-on-a-Chip with Future Artificial Intelligence Integration

Year: $\quad 2019$

Version: Accepted manuscript

Copyright (C) 2019 the authors, published by Wiley. This is the pre-peer reviewed version of the following article: Fetah, K.L., DiPardo, B.J., Kongadzem, E.-M., Tomlinson, J.S., Elzagheid, A., Elmusrati, M., Khademhosseini, A., \& Ashammakhi, N., (2019). Cancer Modeling-on-a-Chip with Future Artificial Intelligence Integration. Small, which has been published in final form at https://doi.org/10.1002/smll.201901985. This article may be used for non-commercial purposes in accordance with Wiley Terms and Conditions for Use of Self-Archived Versions.

\section{Please cite the original version:}

Fetah, K.L., DiPardo, B.J., Kongadzem, E.-M., Tomlinson, J.S., Elzagheid, A., Elmusrati, M., Khademhosseini, A., \& Ashammakhi, N., (2019). Cancer Modeling-on-a-Chip with Future Artificial Intelligence Integration. Small. https://doi.org/10.1002/smll.201901985 


\title{
Cancer Modeling-on-a-Chip with Future Artificial Intelligence Integration
}

\author{
Kirsten Lee Fetah, Benjamin J. DiPardo, Eve-Mary Kongadzem, James S. Tomlinson, \\ Adam Elzgheid, Mohammed Elmusrati, Ali Khademhosseini,* and Nureddin Ashammakhi*
}

\section{Introduction}

Cancer is a leading cause of death worldwide, with an estimated 17.0 million new cancer cases and 9.5 million cancer-related deaths reported by the International Agency for Research on Cancer in 2018. ${ }^{[1]}$ By 2040, global incidence will

K. L. Fetah, A. Khademhosseini, N. Ashammakhi

Center for Minimally Invasive Therapeutics

University of California

Los Angeles, CA, USA

E-mail:khademh@ucla.edu; n.ashammakhi@ucla.edu

K. L. Fetah, A. Khademhosseini, N. Ashammakhi

California NanoSystems Institute (CNSI)

University of California

570 Westwood Plaza, Los Angeles, CA 90095, USA

K. L. Fetah, A. Khademhosseini

Department of Bioengineering

University of California

Los Angeles, CA, USA

B. J. DiPardo, J. S. Tomlinson

Department of Surgery

David Geffen School of Medicine

University of California

Los Angeles, CA, USA

The ORCID identification number(s) for the author(s) of this article can be found under https://doi.org/10.1002/smll.201901985.

DOI: 10.1002/smll.201901985 tumors drastically increase patient mortality and decrease the 34 efficacy of clinical treatments. ${ }^{[4]}$ While patients diagnosed with 35 localized tumors can often be successfully treated with surgery 36 and/or radiation, with relatively high survival rates, a diagnosis 37 of metastatic cancer often designates a terminal illness, with a 38 five-year survival rate less than $20 \%$ for half of all cancer sites. ${ }^{[5]} 39$

E.-M. Kongadzem, M. Elmusrati, N. Ashammakhi

School of Technology and Innovations

University of Vaasa

Vaasa, Finland

A. Elzgheid

Biotechnology Research Center

Libyan Authority for Research, Science and Technology

Tripoli, Libya

A. Khademhosseini, N. Ashammakhi

Department of Radiological Sciences

David Geffen School of Medicine

University of California

Los Angeles, CA, USA

N. Ashammakhi

Division of Plastic Surgery

Department of Surgery

Oulu University

Oulu, Finland 
The development of cancer metastases often necessitates the use of chemotherapeutic drugs, which enter the body's circulation and travel, with inflicting cytotoxicity on tumor cells and therefore impacting tumor growth and, ideally, curing the patient. ${ }^{[6]}$

The staggeringly high mortality and morbidity rates associated with cancer highlight the need for more efficacious therapies. Drug discovery and development for cancer treatment has been slow in its clinical translation due to a high attrition rate during drug development. ${ }^{[7]}$ Despite robust research efforts, only $5.1 \%$ of anticancer drugs that enter phase I clinical trials receive Food and Drug Administration (FDA) approval. ${ }^{[8]}$ One reason for this low translation rate is the poor ability of disease and drug screening models to predict patient outcomes. ${ }^{[7]}$ The improved ability to more accurately and rapidly identify drug candidates and eliminate ineffective drugs as potential candidates would drastically improve the drug development process and accelerate the rate of clinical translation of. ${ }^{[7,9]}$

A central challenge in translating research advances from preclinical models to patient therapies and treatments is the immense complexity of the tumor microenvironment (TME). The TME is a complex niche created by each tumor and influenced by a tumor's interactions. ${ }^{[10,11]}$ It is comprised of the noncancerous cells within a tumor that support tumor cell growth. The TME consists of a heterogeneous population of stromal and immune cells, microvasculature, extracellular matrix (ECM), and the proteins produced and secreted by tumor cells. The TME is characterized by its specific mechanical properties and complex gradients of oxygen, nutrients, and growth factors. These components influence tumor biology and play a role in invasion, metastasis, and treatment outcomes. ${ }^{[12]}$ A comprehensive review of TME components' respective roles in these processes and cancer treatment can be found in previously published review papers. ${ }^{[10,13]}$ Current preclinical models for anticancer drug screening fall into two categories, in vitro and in vivo models. The simplest, and most commonly used, in vitro model is the traditional $2 \mathrm{D}$ culture of immortalized cell lines. ${ }^{[14]} 2 \mathrm{D}$ culture is relatively inexpensive and allows for high-throughput analysis and is therefore commonly used in studies aiming to elucidate cancer biology or identify novel chemotherapeutic agents. However, despite their advantages, these models subject cancer cells to artificially 2D growth conditions and lack key components of the TME that influence cancer biology and drug response, including the stroma, ECM, tumor mechanical properties, and intertumoral gradients. ${ }^{[14,15]}$ As a result, the predictive value of these models is limited. Another traditional preclinical tool for cancer studies is in vivo animal models. Animal models for cancer mimic the tumor microenvironment to a greater fidelity than simplified 2D cell culture. ${ }^{[16-18]}$ These models are often used to study an individual patient's tumor ex vivo via patient-derived xenograft (PDX) models. However, despite their advantages, these models have several limitations. While these models provide a 3D environment, a comparison between two vastly different species cannot be made with high accuracy. As a result, there is often a disparity between the outcomes of cancer related drug studies in animal models and patient trials. In addition, animal studies are often cost prohibitive. Thus, an alternative model is needed to provide efficient and effective drug screening to guide the

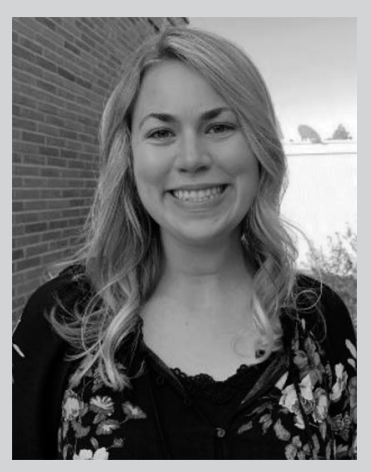

Kirsten Fetah is a graduate student in the Department of Bioengineering at UCLA. Her graduate school research is focused on biomaterials for tissue engineering and miniaturized tissue systems for personalized medicine. Previously, she worked at UC Berkeley in the field of mechanobiology, using synthetic biology techniques to control cytoskeletal contractility and study how cells sense the mechanical properties of their environment. Kirsten is interested in collaborating with clinical partners through her work at UCLA in order to translate research done in the lab into new therapeutic solutions.

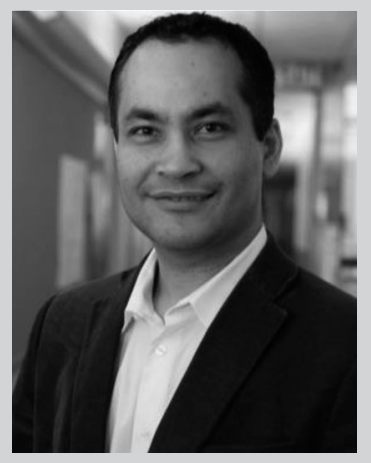

Ali Khademhosseini is the Levi Knight Professor of Bioengineering, Chemical Engineering and Radiology at UCLA. He is the Founding Director of the Center for Minimally Invasive Therapeutics at UCLA. Previously, he was a professor of medicine at Harvard Medical School. His research interest is focused on combining micro- and nanoengineering approaches with advanced biomaterials for regenerative medicine applications.

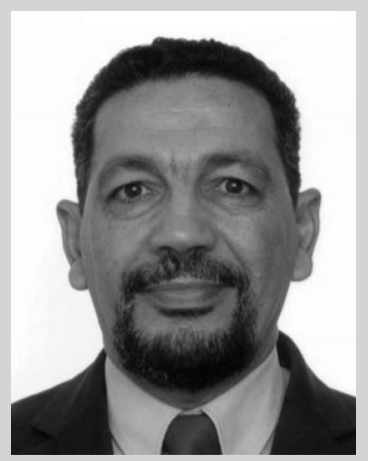

Nureddin Ashammakhi is an associate director of the Center for Minimally Invasive Therapeutics at UCLA, leading translational research in regenerative therapy. He has extensive experience with biodegradable implants, drug release, and nanofiberbased scaffolds. Currently, he is working on 3D bioprinting and organ-on-a-chip models for regenerative and personalized medicine. He was previously a professor of biomaterials technology in Tampere University of Technology, Finland, Chair of Regenerative Medicine in Keele University, UK and Adjunct Professor in Oulu University, Finland before he joined University of California - Los Angeles first as a visiting professor (scholar) and then as a faculty. 
initial selection of chemotherapeutic agents for cancer patients. This need drives the development of 3D models, which seek to integrate the advantages of in vivo and in vitro techniques for improved cancer studies and drug development.

Recently, much effort has been put into the development of organ-on-a-chip platforms that recapitulate both the biology and physiology of in vivo human tumors. ${ }^{[7,19-21]}$ The majority of these platforms are designed to mimic crucial functions of organs and tissues, enabling the investigation of pharmacokinetics and pharmacodynamics. ${ }^{[19]}$ These approaches employ the use of multichannel, 3D microfluidic chips to simulate the mechanics, activity, and physiological response of organs. Cancer-on-a-chip models are at the frontier of nanomedicine and offer promising utility as sophisticated microsystems to elucidate the mechanisms of cancer biology and improve anticancer drug development. As new organ-on-a-chip platforms are developed, becoming increasingly higher throughput, large data sets are generated, bringing forth new challenges and opportunities. The development of these high-throughput organ-on-a-chip platforms gives rise to the application of deep learning-based analysis processes for high-throughput drug screening, making way for a new avenue of cutting-edge research. ${ }^{[22]}$ In this review, we will highlight seminal canceron-a-chip papers and new advances in the field, incorporating discussion on key features of the TME and the challenges associating with recapitulating its features. We will discuss recent applications of cancer-on-a-chip models for pathomorphological and drug development studies, with special emphasis on the integration of and challenges associated with combining these platforms with machine learning (ML) and data processing technologies. Future perspectives on how cancer-on-a-chip and machine learning algorithms can synergize to improve anticancer drug development will be considered.

\section{Anticancer Drug Development and the Need for Improved Predictive Models}

The anticancer drug development process starts with the identification of effective compounds via preclinical models. These compounds are then evaluated further in sequential human clinical trials to assess safety, dosing, and efficacy, comparing the drug in question to the current standard of care. Unfortunately, the majority of compounds identified as effective in preclinical models are found to be not safe or efficacious in these later studies. ${ }^{[18,23]}$ The low predictive value of preclinical models increases the cost and resources expended during the drug development process. Such clinical trial failures point to the need for improved preclinical models that mimic the TME with high fidelity. ${ }^{[1,18,23]}$

In addition to the challenges associated with current preclinical models, progress in anticancer drug development is hindered by the complexity of cancer biology. Cancer biology is highly heterogeneous and complex on several levels: among tumor subtypes, individual patients, and separate tumors within one patient. Selection of chemotherapeutic agents is currently dominated by evidence generated from randomized clinical trials (RCTs), in which patients are assigned to treatment groups by chance. Given the ongoing development of many new chemotherapeutics and the necessity to monitor the stability of 1 previously developed agents, it is impossible to perform RCTs 2 to investigate all FDA-approved drugs for each tumor type, let 3 alone trials to investigate all drug combinations. An individual 4 patient's cancer biology is unique and tumors of the same his- 5 tologic subtype often show vastly different responses to thera- 6 pies. Thus, initiatives for precision, or personalized, medicine 7 have emerged. ${ }^{[24]}$ This emerging field is based on the principle 8 that the genetic and molecular information of an individual 9 patient can be used to deploy more effective, less toxic, and 10 patient-specific treatments. ${ }^{[24]}$ Furthermore, in order to person- 11 alize the chemotherapy regimens given to a patient and opti- 12 mize the chance of an effective response, there exists the need 13 to develop robust models to perform drug screening for both 14 general cancer subtypes and for an individual patient's tumors. 15

\section{Comparison of Preclinical Cancer Models}

\subsection{D Monoculture}

21

In traditional 2D cultures, cells are grown as an adherent mon- 22 olayer in a culture dish, attached to the plastic dish surface. ${ }^{[25,26]} 23$ Assays derived from 2D monolayers are easy to use, low cost, 24 and high throughput. However, despite their advantages, these 25 assays are quite limited in their predictive value. One such 26 limitation is the inability of 2D cell cultures to mimic the native 27 structure of tissues and tumors. ${ }^{[14,26]}$ The 2D culture environ- 28 ment does not recapitulate the cell-cell and cell-environment 29 interactions present in native tumor. ${ }^{[14,26]}$ These interactions are 30 fundamental to cell proliferation, cell differentiation, gene and 31 protein expression, stimuli response, drug metabolism, and 32 other cellular functions. ${ }^{[14,27]}$ Another limitation of 2D culture 33 models is that cells in an adherent monolayer have infinite, 34 homogenous access to key nutrients, including oxygen and 35 metabolites. ${ }^{[14,26]}$ In vivo, cancer cells have more variable access 36 to nutrients and oxygen due to natural tumor architecture. ${ }^{[27]} 37$ Because of these disadvantages, there exists the need to 38 find alternate models which better mimic the native cancer 39 microenvironment.

\subsection{Transwell Model}

$\begin{array}{ll}43 & 44\end{array}$

Transwell assays are used to study the invasion and migrations 45 of cancer cells. ${ }^{[28]}$ These assays use a cell culture insert made 46 of a porous, polymeric membrane that allows for migration 47 through the pores. ${ }^{[29,30]}$ Transwell assay applications include 48 migration, invasion, and transendothelial migration. ${ }^{[29,30]} 49$ Migration assays, the simplest transwell-based assay, seed 50 cancer cells on top of the polymeric membrane insert and 51 measure the ability of the cells to translocate though the mem- 52 brane's pores. ${ }^{[28-30]}$ Invasion assays are more complex in that 53 they add a layer of ECM on top of the porous membrane and 54 characterize cancer cell migration through the ECM. ${ }^{[28-30]}$ Tran- 55 swell assays are used as both a tool for drug screening and a 56 model for studying cancer cell migration, invasion, extravasa- 57 tion, and matrix remodeling. The transwell-based assay serves 58 as a straightforward in vitro technique for studying a tumor's 59 
ability to metastasize to a secondary site. However, despite their advantages, transwell assays study the motility of individual cells and, as a result, is not an optimal tumor model.

\subsection{D Culture Models}

3D culture models use various matrices or scaffolds for cancer cells to grow and ECM, thus mimicking important components of the TME. Scaffolds support cell attachment, growth, and morphogenesis. ${ }^{[29]}$ These scaffolds are typically made from natural or synthetic materials, such as gelatin, collagen, alginate, hyaluronic acid, polyethylene glycol, or polylactide, polylactide-co-glycolide, and various other polymers. ${ }^{[7]}$ These scaffold-based approaches are ideal in that they have similar mechanical and physical properties to the native ECM and TME. ${ }^{[7]}$ More recently, alternative approaches to scaffolds have been developed, including the creation of cell spheroids via bioprinting. ${ }^{[7]}$ These structures have improved perfusion due to their vascularization but are limited by technical challenges and their capacity to recapitulate complex tissue types. ${ }^{[21]}$ Various other methods have been developed, for example, the use of nonadhesive polyethylene glycol di-methacrylate hydrogel microwells to produce cancer cell multicellular aggregates, ${ }^{[31]}$ hanging drop cultures, and spinner cultures. While these methods can be improved upon, these models are an important foundation for more novel platforms, such as cancer-on-a-chip, as they model tumor-tumor cell interactions, native ECM, and may be designed to recapitulate the biophysical properties of native tumor.

\subsection{Animal Models}

Preclinical animal models are a necessary component in the process of anticancer drug development and discovery. ${ }^{[32]}$ These in vivo models capture physiological complexity with higher fidelity than 2D monoculture techniques. ${ }^{[7]}$ While these models have vastly improved our understanding of cancer, they are also limited in their capacity. One such shortcoming of animal models is their limited translatability to humans. The inability of animal models to fully recapitulate human cancer physiology is evidenced by the failure in clinical trials of drugs identified in preclinical results. ${ }^{[7]}$ To improve the replicative value of in vivo animal studies, PDX tumor models have been established. PDX models are created by implanting cancer cells or tissues derived from patient tumors into immunodeficient mice. ${ }^{[3]}$ PDX models are used extensively in cancer research, as they simulate human tumor biology in vivo. While these models better replicate human tumor biology, the use of immunocompromised animals impedes analysis of the immune system's response to a tumor. ${ }^{[17,34]}$ An additional challenge with PDX models is the establishment rate. Previous research reports a successful formation rate of implanted tumors as being $39.2 \% .^{[35]}$ As animal models are expensive, highly regulated, and limited by a low initiation rate, there are constraints to the number of studies that can be done, preventing the PDX model from being a high-throughput assay. In addition, the procedure of creating PDX models takes months to establish, making PDXs logistically difficult for use in making timely 1 clinical decisions. ${ }^{[36]}$ Despite their advantages, animal models 2 do not practically allow for the high-throughput assessment of multiple combinations of chemotherapeutics, highlighting further the need for high-throughput platforms to be used in 5 precision medicine to identify anticancer drugs on a patient- 6 specific basis. A thorough discussion of preclinical models and 7 their advantages and disadvantages can be found in previously published reviews. ${ }^{[14,26,28,37]}$

\section{Cancer-on-a-Chip Platform}

\subsection{Organ-on-a-Chip Structure and Function}

In recent years, organ-on-a-chip platforms have significantly advanced for several applications, such as preclinical drug screening and disease modeling. Organs-on-a-chip are microdevices with miniaturized tissues to model human organ physiology in vitro. ${ }^{[38-41]}$ Organ-on-a-chip devices incorporate microfluidics with $3 \mathrm{D}$ tissues to recapitulate native organ complexity and cues, for example, electrical signals, fluid flow, and biochemical cues. Organ-on-a-chip has many advantages, including improved recapitulation of native microenvironment, simplicity, decreased cost, and reproducibility. The microfluidic chip components are traditionally made of polydimethylsiloxane (PDMS), which has ideal properties, such as transparency, low toxicity to cells, and high permeability to $\mathrm{O}_{2}$ and $\mathrm{CO}_{2}$ gases. ${ }^{[39]}$ Cells are cultured in small chambers within these miniature chips, either in 2D monolayers or 3D suspensions, to emulate organ tissues. Membranes may be integrated into the microfluidic chips, creating multiple channels and separating the cells. ${ }^{[40-47]}$ The microfluidic components of organ-on-a-chip platforms recapitulate in vivo conditions, such as flow, pressure, and nutrient levels. Organs-on-a-chip are also able to expose the cells or tissues to controlled laminar flow of fluids, improving the accuracy of biomarker identification and drug screening. ${ }^{[48]}$ Examples of organ-on-a-chip platforms include thrombosis-on-a-chip, ${ }^{[49]}$ alveolus-on-a-chip, ${ }^{[50]}$ lungon-a-chip, ${ }^{[45]}$ and gut-on-a-chip. ${ }^{[45]}$ More details of organ-on-achip development and applications can be found in previously published reviews. ${ }^{[38,51,52]}$

\subsection{Organ-on-a-Chip Advantages}

Organ-on-a-chip platforms are miniaturized, reducing the required sample sizes and materials consumed during in vitro testing. ${ }^{[53]}$ As a result, organ-on-a-chip testing is less costly than alternative preclinical models, such as animals. In addition, organs-on-a-chip perhaps offer an ethical advantage and alternative to animal models. Due to the miniaturized nature of these platforms, it has become possible to use materials from a single animal to run hundreds of tests, instead of running a single test on hundreds of animals. The small size and low cost of organs-on-a-chip allow for accelerated research and testing, as many samples can be run on one device. Another advantage of organs-on-a-chip is the ability to recapitulate native microenvironments, modeling mechanical stresses, nutrient diffusion,

1
2
3
4
5
6
7
8
9


and fluid flow, for example. These advantages have sparked interest in combining organ-on-a-chip platforms with other 3D models, such as organoid cultures. While 3D organoids may recapitulate a singular organ, organ-on-chip platforms mimic how these organs interact with their in vitro environment. Further discussion on the combination of 3D organoid cultures with organs-on-a-chip can be found in recent reviews. ${ }^{[53,54]}$

\subsection{Cancer-on-a-Chip Platforms}

Cancer-on-a-chip platforms were developed by using cancer and tumor-derived cells and matrix materials inside of previously developed cancer-on-a-chip platforms. The microfluidic devices developed for organ-on-a-chip platforms have shown to be promising for applications in large-scale, high-throughput anticancer drug screening, study of metastatic cancer processes, and screening of drugs against a patient's individual tumor (personalized medicine)..$^{[7,13,21,55]}$ In the subsequent sections, we will focus our discussion on cancer-on-a-chip models that recapitulate components of the TME. A discussion of canceron-a-chip to evaluate nanomedicine ${ }^{[19]}$ and for personalized medicine applications ${ }^{[56]}$ can be found in recently published reviews.

\section{Applications of Cancer-on-a-Chip Technologies}

The development of cancer-on-a-chip systems has greatly expanded the ability of in vitro models to recapitulate the TME. With the recent development of dynamic culture systems and the advent of organ-on-a-chip platforms, which offer spatial and temporally controlled microenvironments, the improved cancer modeling has become an attractive prospect for studying both cancer biology and treatment options. ${ }^{[57]}$ Although this field is still in its infantile stage, its progress is expected to grow exponentially. Cancer-on-a-chip models offer many advantages. First, they allow researchers to mimic elements of the TME in isolation or in concert, including the addition of cancer cells to stromal cells, immune constituents, vasculature, and oxygen, nutrient, or growth factor gradients. Cancer-on-a-chip systems also allow for noninvasive real-time monitoring of crucial cellular parameters and recapitulate the complex cellular and extracellular microenvironment of tumors. These abilities allow for the investigation into the role microenvironmental features play in the progressing stages of cancer metastasis. ${ }^{[24]}$

Cancer-on-a-chip models have been used to evaluate aspects of cancer biology in several different malignancies, examining local tumor invasion, metastasis and angiogenesis, as well as serving as models for immunotherapy research and drug screening. ${ }^{[4,7,29]}$ These studies examined several types of cancer as model systems, including some of the most common and deadly cancers, for example, breast, ${ }^{[36,58,59]}$ lung, ${ }^{[20,60]}$ and pancreatic cancer. ${ }^{[15]}$ It is very important to work on these frontiers, but, nevertheless, other cancer models should be developed both to address special characteristics and the subtypes of cancers, as well as the comprehensive spectrum of cancers, including those of the head and neck.
5.1. Cancer-on-a-Chip Systems Model Tumor Morphology and Drug Response with High Fidelity

Cancer-on-a-chip systems that recapitulate in vivo cancers and 4 tumors are fundamental to improved strategies for anticancer 5 drug selection. In 2014, Vidi et al. developed a cancer-on-a-chip 6 model, mimicking cancer mammary ducts. ${ }^{[61]}$ The breast-on- 7 a-chip device was comprised of a breast luminal epithelium 8 monolayer on a semicircular acrylic support. Tumor cells grown 9 in these channels were different morphologically than the same 10 cells cultured on a traditional flat surface. Additionally, tumor 11 nodules cultured in these channels displayed a different anti- 12 cancer drug sensitivity compared to their flat and monoculture 13 counterparts, providing new insight for the design and testing 14 of cancer therapies.

An important component of developing a new platform is 16 the comparison of its results to the existing standard. In 2017, 17 Beer et al. compared their pancreatic ductal adenocarcinoma- 18 on-a-chip model to other in vitro and to in vivo PDX models 19 (Figure 1). ${ }^{[15]}$ Their HepaChip consisted of eight chambers, 20 each containing three $1 \mathrm{~mm} \times 60 \mu \mathrm{m}$ cell culture regions, 21 which were coated with collagen. These cell culture regions 22 were irradiated by UV light, creating acid groups to which 23 collagen was bound. Electrodes were integrated on the wall 24 of each chamber, creating dielectrophoretic forces. Pancre- 25 atic ductal adenocarcinoma (PDAC) human cell lines were 26 cultured inside of the polymer chambers. The combination 27 of microfluidics and dielectrophoresis assembled in vitro 28 micro-organs. The experimental results show morphological 29 and growth characteristics more like that of spheroid cultures 30 than the 2D culture. Compared to traditional 2D preclinical 31 platforms, the HepaChip model is better at capturing cell-cell 32 and cell-ECM interactions, and thus it is more biomimetic, 33 representing a more predictive model with potential to be 34 useful in the development of personalized pancreatic cancer 35 treatment.

Another more recent study by Lanz et al. utilized breast 37 cancer cell lines for developing a high-throughput breast- 38 cancer-on-a-chip to study the response of triple negative 39 breast cancer cell lines (MDA-MB-453, MDA-MB-231, 40 AND HC1937) to anticancer therapeutic drugs (paxlitaxel, 41 olaparib, and cisplastin). ${ }^{[36]}$ Several conditions were evalu- 42 ated, including cell seeding density, ECM composition, 43 biomechanical conditions, and the response to therapy, as 44 compared to those seen in 2D cultures. ${ }^{[36]}$ Differences in drug 45 response were observed in different ECM materials (Matrigel 46 vs BME2rgf vs collagen 1). This microfluidic platform allowed 47 for the simultaneous culture of 96 perfused microtissues 48 $(\approx 10$ cells per data point). Additional advantages include the 49 use of small quantities of material and the ability to perform 50 drug screening using patient-derived samples. This strategy 51 is a vast improvement from previous $3 \mathrm{D}$ culture techniques, 52 as it allows for constant perfusion of the culture medium. 53 While the tested system did not entirely capture in vivo com- 54 plexities, this strategy presents a more high-throughput and 55 efficient system for testing and raises the possibility for use 56 in developing personalized medicine by determining appro- 57 priate drug sensitivity and predicting individual patient 58 response in a real-time fashion.

10

1

(1)

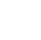


A

B

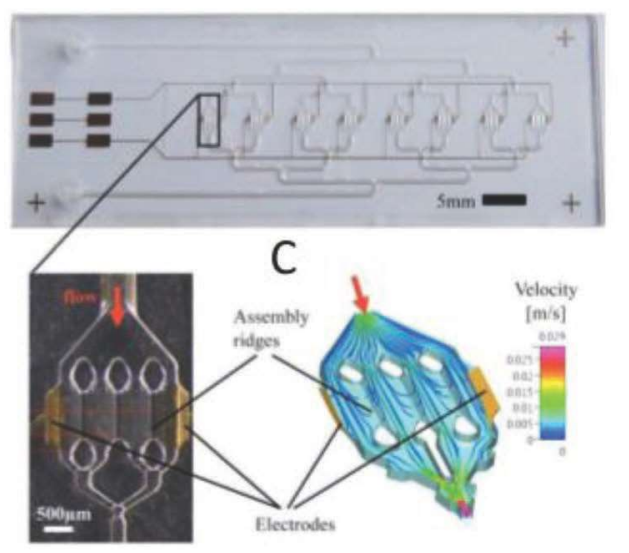

D
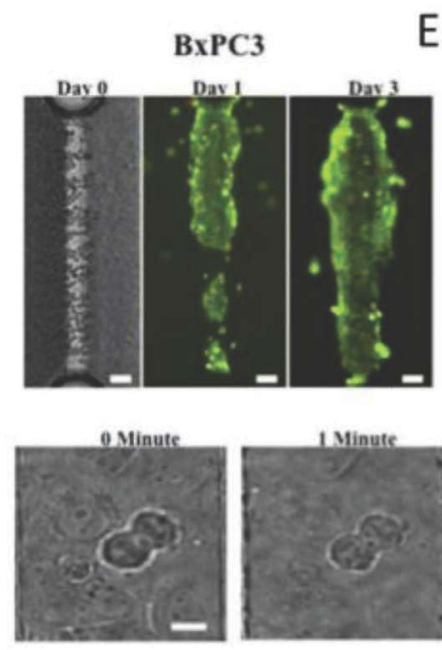

$\mathrm{E}$
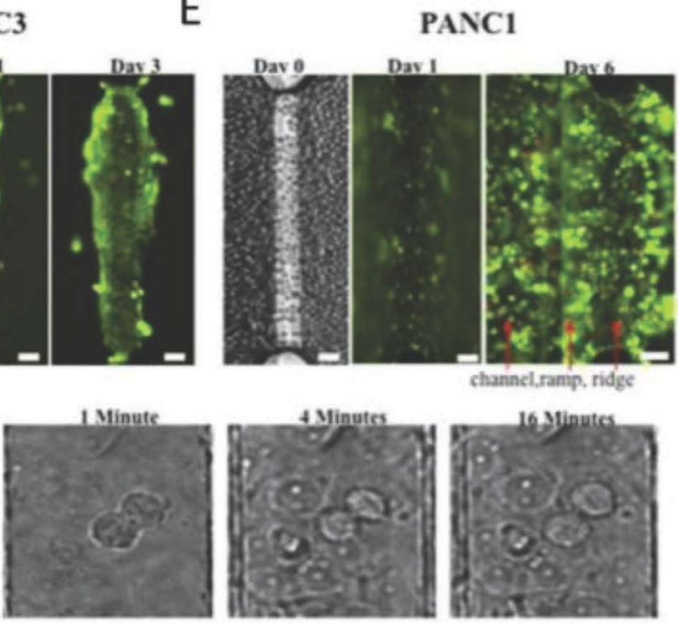

Figure 1. Photographs and simulation of the HepaChip. A) Image of the chip, with eight culture chambers, fluid inlet and outlet, and gold electrodes. B) Enlarge view of single chamber, with two electrodes and three assembly ridges. C) Simulation of the flow and cell trajectory inside of the culture chamber. D) Live/dead staining of BxPC3, growing on the assembly ridge. E) Live/dead staining of PANC1, spread on well channel walls and bottom F) Mitosis of MxPC3, observed after $16 \mathrm{~h}$ culture. Reproduced under the terms of the Creative Commons Attribution 4.0 International License. ${ }^{15}$ Copyright 2017, The Author(s). Published by Springer Nature.

\subsection{Cancer-on-Chip Systems Model Mechanical Properties of the Tumor Microenvironment}

In addition to modeling tumor morphology and drug response, cancer-on-a-chip platforms also offer improved modeling of the TME's mechanical properties, such as its stiffness, which plays a role in cancer progression. ${ }^{[62]}$ In 2017, Hassell et al. studied non-small-cell lung cancer (NSCLC) in an organ-on-a-chip device (Figure 2) ${ }^{[60]}$ This NSCLC-on-a-chip model recapitulated organ microenvironment-specific growth, tumor dormancy, and response to tyrosine kinase inhibitor (TKI), a therapy used in vivo in human patients. This new platform revealed a newly observed mechanical sensitivity of NSCLCs. TKI therapeutic response was discovered to be sensitive to the physical cues of breathing motions, with mechanical breathing motions perhaps suppressing NSCLC response to TKI therapy. This new finding elucidates understanding of NSCLC and can help to explain therapy resistance in patients with lung tissue that remains aerated and mobile, as mechanical strain leads to the downregulation of epidermal growth factor receptor (EGFR), which is partly responsible for decreased response to therapy in persistent tumors. ${ }^{[60]}$ The understanding of this previously unstudied mechanism has implications in future mitigation of drug resistance and development of efficacious therapies.

These findings further validate the need for the development of dynamic systems and platforms, which take into consideration the components of the TME. ${ }^{[63]}$ Future work need not only consider traditional properties, such as cell type and ECM, but 2 also dynamic properties, such as mechanical stiffness, to mimic 53 in vivo events and develop more reliable predictive models.

54

55 5.3. Cancer-on-Chip Systems Model Tumor Immune

56 Microenvironment

57

58 Immune system elements play a large role in the TME, 59 with crosstalk between the immune and cellular TME components. ${ }^{[64]}$ Interactions within the TME contribute to 24 cancer initiation (carcinogenesis), progression, and metastasis. 25 After a tumor escapes immune recognition, the TME affects 26 immune cell behavior and the two play a synergistic role in 27 tumor progression. ${ }^{[65]}$ Organ-on-a-chip platforms can be useful 28 in the development of immuno-oncology models. Such a model 29 was shown by Biselli et al. in their work on mononuclear and 30 cancer cells (Figure 3). ${ }^{[66]}$ The presented cancer-on-chip model 31 studied the crosstalk between immune cells, leukocytes, and 32 human breast cancer cells treated with chemotherapeutics. ${ }^{[76]} 33$ The study compared leukocytes with and without the FRP1 34 gene and concluded that leukocytes lacking the FPR1 gene 35 do not recognize the chemotherapy-treated cells, while leuko- 36 cytes with expression of FPR1 perform random walks, drifting 37 toward the tumor cells and establishing persistent interac- 38 tions with them. ${ }^{[66]}$ These findings demonstrate the capacity 39 and necessity to develop immune-oncology methods using 40 the organ-on-a-chip platform, as immune response is a key 41 component of the in vivo environment (evidenced by the cell- 42 cell interactions of immune and tumor cells). ${ }^{[66,67]}$ A detailed 43 discussion on crosstalk between the TME and immune system 44 can be found in a recent review paper. ${ }^{[64]}$

\subsection{Cancer-on-Chip Systems Model Tumor Angiogenesis, 48} Microvasculature, and Lymphatics

Angiogenesis plays a critical role in both the growth and meta- 51 static spread of tumor cells. ${ }^{[68,69]}$ The TME can regulate angi- 52 ogenesis, via ECM molecules and growth factors within the 53 TME. ${ }^{[70]}$ Increased vascularity has been observed, even in the 54 bone marrow, in patients with hematological malignancies. ${ }^{[68]} 55$ Despite this observation, the role of angiogenesis in regulating 56 hematological malignancies is not well understood. ${ }^{[68]}$ In 2016, 57 Zheng et al. used a 3D microfluidic angiogenesis-on-a-chip 58 to study the unique morphogenic signatures of angiogenesis 59 
A

C

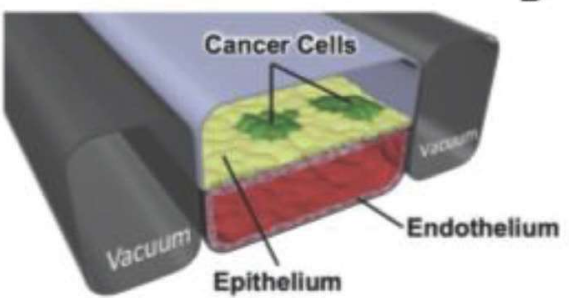

B
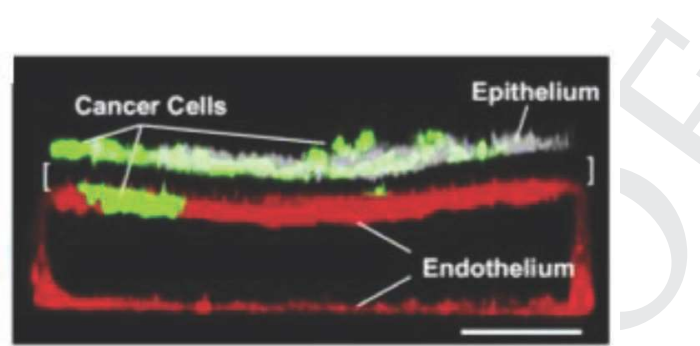

\section{Day:}

E
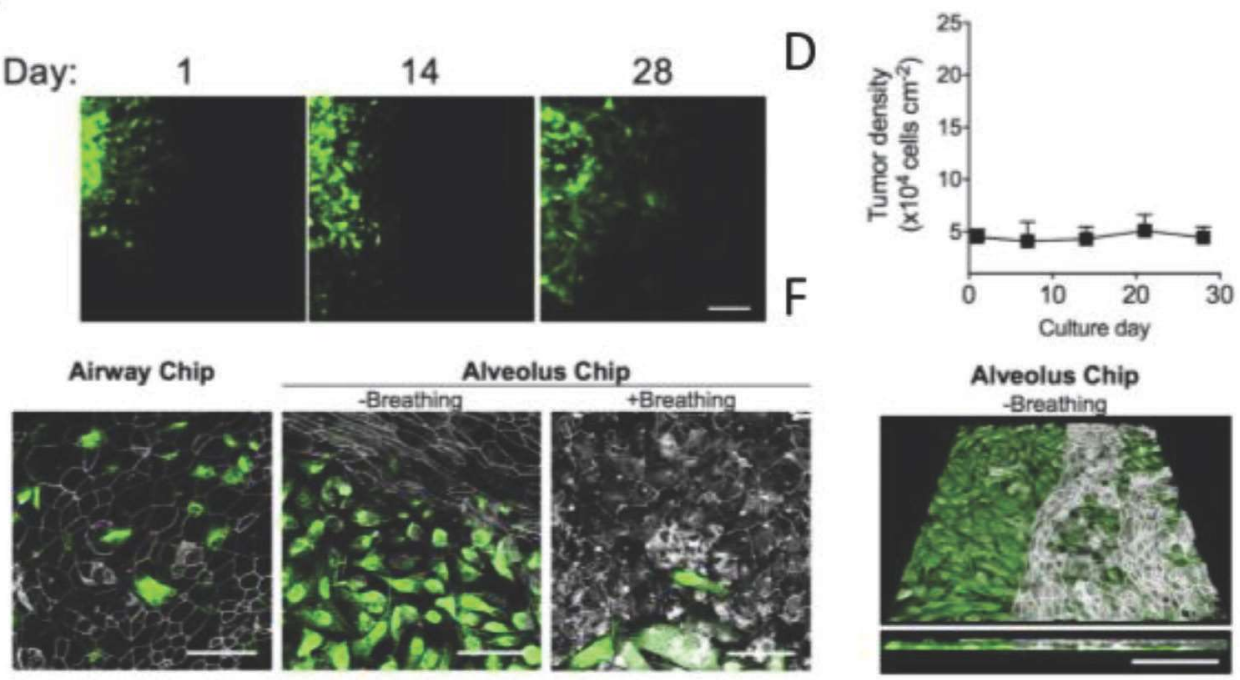

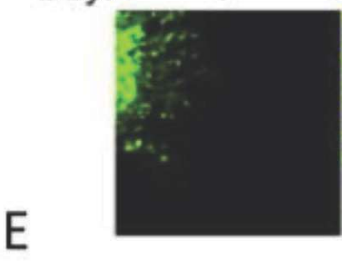

2

3

4

5

6

7

8

9

10

11

Figure 2. Human orthotopic lung cancer-on-a-chip model. A) Schematic of a cross section through the designed microfluidic chip. B) Microscopy 29 image of a cross section of the two central channels of the alveolus chip taken via fluorescence microscopy image. C) Immunofluorescence microscopy 30 image of a cluster of GFP labeled NSCLC cells, implanted in the airway chip. D) Quantification of NSCLC densities after implantation in the chip. 31 E) Growth pattern of GFP labeled lung cancer cells within the epithelial monolayer. F) Lung cancer cell growth dynamics. Reproduced with permission. ${ }^{[60]} 31$ Copyright 2017, Elsevier.
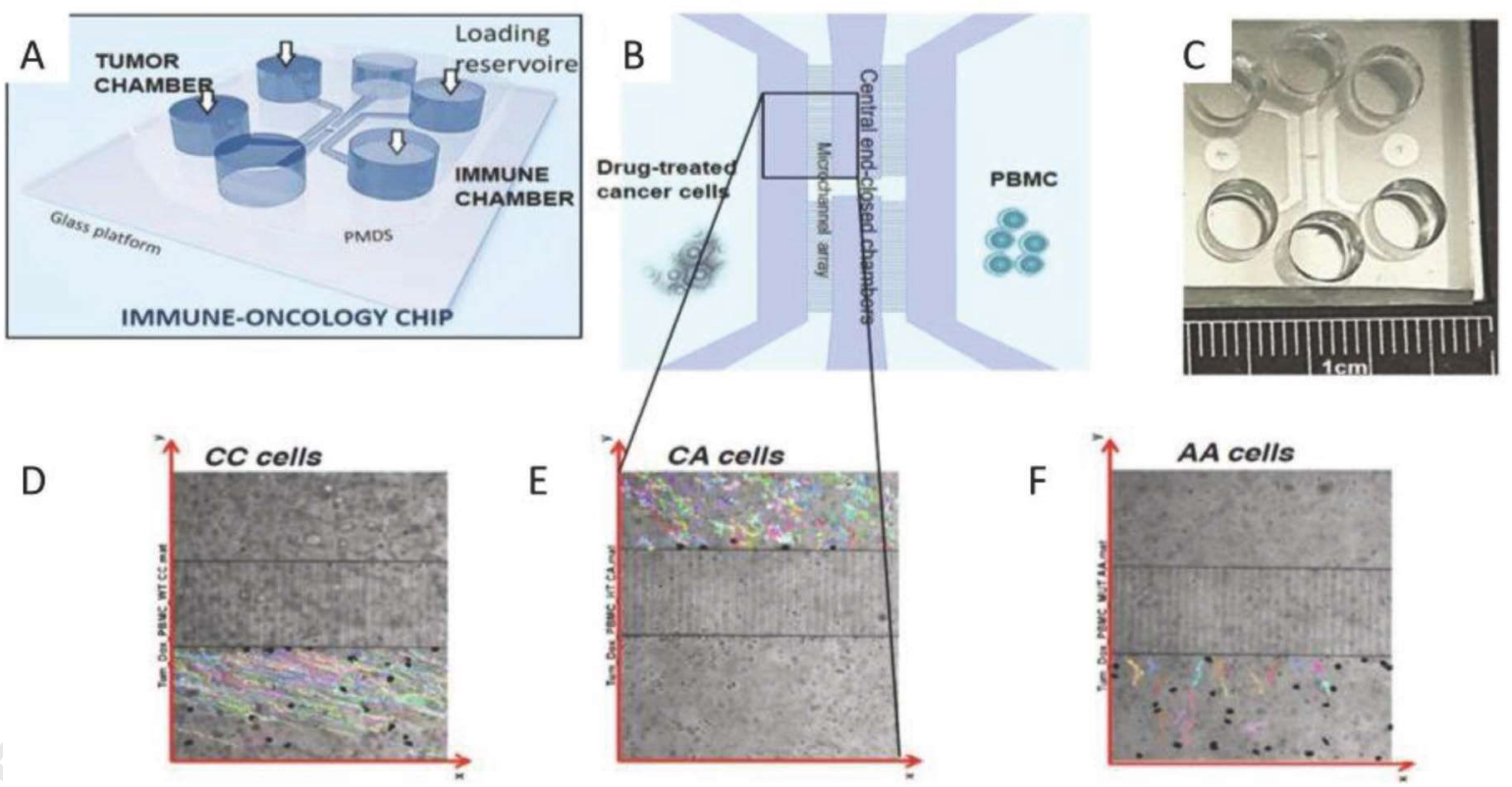

D

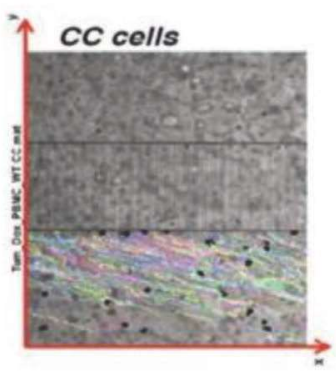

$E$

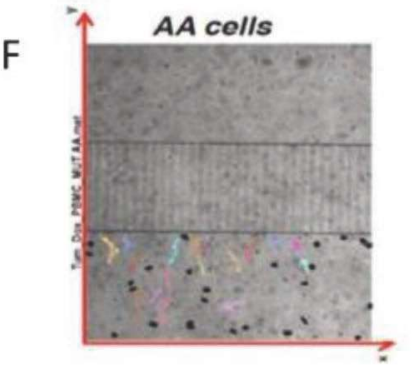

Figure 3. A) General schematic of the immune-oncology chip, whose design features six reservoirs for cells loading and culture medium replacement and four compartments for cell culture. B) Detailed view of the four chambers. C) Picture of the whole device. D-F) Trajectories of FPR1 CC cells, FPR1 CA cells, and FPR1 AA cells, respectively. Reproduced under the terms of the Creative Commons Attribution 4.0 International License. ${ }^{[66]}$ Copyright 2017, The Author(s). Published by Springer Nature. 
induced by leukemic cells, with or without bone marrow stromal cell coculture. ${ }^{[68]}$ The microfluidic device was fabricated from PDMS by soft lithography, forming three parallel microchannels, separated by trapezoidal posts. The central channel was filled with collagen 1 and the side channels with endothelial and leukemia derived cells. The role of leukemic cells on angiogenic induction and vessel formation was observed. This model has not yet been achieved via existing 2D culture techniques. Angiogenesis in the bone marrow is a highly dynamic process and is critically dependent on both cell-cell and cellmatrix interactions, which are abundant in the native bone marrow microenvironment, presenting the critical need for functional angiogenic assays.

The lymphatic system serves as a method through which many cancers can be disseminated. New microfluidic flow systems make it possible to study the role of lymphatic capillary microenvironment in the lymphatic invasion of mammary adenocarcinoma cells. ${ }^{[69]}$ This platform allows for the quantification of cell transmigration and its dynamics, revealing that both luminal and transluminal flow are important in increasing tumor transmigration, as opposed to the previous belief that this behavior was a result of luminal flow alone. This study provides new insights on flow-mediated regulation of lymphatic tumor migration and presents a new tool for exploration of cancer therapy, allowing for medium-to-high throughput studies.

\subsection{Cancer-on-Chip Systems Model Cancer Invasion and Metastasis}

Cancer metastases contribute to over $90 \%$ of cancer-related mortalities. ${ }^{[71]}$ Thus, the use of experimental models to effectively represent the metastatic microenvironment is warranted. Metastasis-on-a-chip platforms allow for the study of important aspects of the metastasis process, such as physiochemical factors from the tumor stroma and heterocellular interactions, which influence cell migration, as well as physicochemical gradients, which lead to tumor cell motility and invasion. ${ }^{[21]}$ An early study to visualize metastatic progression in 2007 by Yates et al. developed a system to visualize the interactions between tumor cells and target organs to where they metastasize. ${ }^{[72]}$ This study investigated hepatic cells with prostate and breast carcinoma cells, examining tumor cell invasion and expansion. They found that tumor cells were unable to grow without a supporting hepatic microtissue, due to the absence of paracrine functionality or liver structure support. The developed system served as a crucial model for examining tumor-host interactions during the processes of metastasis and invasion, circumventing the limitations of previous models.

More recently, microfluidic metastasis-on-a-chip models have been designed to more accurately study cancer progression. ${ }^{[59]}$ These studies investigate the various stages of cancer metastasis, lymphangiogenesis, ${ }^{[73]}$ and angiogenesis, intravasation, ${ }^{[74,75]}$ arrest, organ-specific extravasation, ${ }^{[76-79]}$ and the formation of micro-metastases. Additionally, studies looked at invasion rate as a method for studying cancer. ${ }^{[80]}$ In 2018 , Hao et al. developed a bone-on-a-chip model as aid in the study of breast cancer metastasis to bone tissue. ${ }^{[58]}$ This new bone-on-chip design is miniaturized, increasing experimental 1 throughput, and facilitates easy and frequent observations. 2 Fundamental markers of breast cancer colonization of the bone 3 were observed and confirmed with in vivo collected data.

Another organ-on-a-chip study constructed a multiorgan 5 microfluidic chip platform to investigate lung cancer metas- 6 tasis. ${ }^{[20]}$ This multi-organ-on-a-chip system consisted of four 7 organs, one upstream lung and three downstream organs, 8 with three parallel microchannels formed by PDMS. Bronchial 9 epithelial, lung cancer, microvascular endothelia, and fibroblast 10 cells were grown in the lung organ, and astrocytes, osteocytes, 11 and hepatocytes were grown in the three downstream organs, 12 mimicking the metastasis of lung cancer to the brain, bone, 13 and liver. Damage to the astrocytes, osteocytes, and hepatocytes 14 validated metastasis in the organs-on-a-chip system.

Migration and invasion studies in cancer-on-a-chip models improved upon the use of more traditional assays, such as transwell cultures and scratch-wound assays. Recent work from Toh et al. utilized a microfluidic cancer-on-chip cell migration model which resolved different aspects of cell intravasation (the invasion of cancer cells into a blood or lymphatic vessel) in a biologically relevant 3D microenvironment. ${ }^{[81]}$ The described platform incorporates a 3D microenvironment, which plays a critical role in the invasive properties of cancer cells, with a microfluidic system, creating an appealing model for the testing of antimigration and anti-invasion cancer drugs, which can be multiplexed to allow for high-throughput assays. The development of new tumor models will be crucial in improving management of cancers and the prognosis of cancer patients and, as a result, may help ultimately in the reduction of healthcare costs. ${ }^{[82]}$

\subsection{Cancer-on-Chip Systems for Use in Chemotaxis Studies}

The cell types and factors in the TME influence the occurrence of cancer migration modes. ${ }^{[83]}$ Microfluidic organ-on-a-chip systems have been used to study the chemotaxis, or migration due to chemical gradients, of cancer cells. ${ }^{[84]}$ Aung et al. developed a cancer-on-chip platform with cancer spheroids encapsulated with gelatin methacryloyl (GelMA) hydrogel, surrounded by an endothelial cell barrier. ${ }^{[84]}$ For this study, they harnessed the chemoattracted-induced motility of human umbilical vein endothelial cells (HUVEC) and cancer spheroids to control organization within the microfluidic device. Cancer cell migration was observed in relation to the presence and location of the chemotactic source. Although this study uses an endothelial cell-cancer cell coculture, this approach provides a framework for establishing platforms with the same level of complexity as physiological tumors. These models bridge the gap between in vitro cell culture and in vivo animal experiments and serve as a promising platform for studying tumor behaviors in the vascular system. ${ }^{[85]}$ More complex, hybrid cancer-on-a-chip models incorporate 3D tumor tissue models, such as spheroids, resulting in more advanced and highperformance models. ${ }^{[86,87]}$ In the future, these models will have elucidated mechanisms for cancer invasion, such as chemotaxis, and present the possibility of developing migration inhibitory drugs. ${ }^{[84]}$

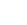
.

.

0

1
12 


\subsection{Cancer-on-a-Chip Platform for Cancer Treatment and Drug Development}

Improved 3D culture models have been developed with the prospect of accelerating the selection of therapies by improving the ability to predict anticancer drug responses. ${ }^{[36]}$ One avenue of new cancer-on-a-chip studies involves the use of 3D microfluidic devices for improved anticancer drug screening and selection. Examples of these new strategies include a 3D high-throughput perfused microfluidic platform for testing new breast cancer therapies, developed by Lanz et al. (Figure 4), ${ }^{[36]}$ and microfluidic platform for studying biomolecular characteristics of pancreatic ductal adenocar- 1 cinoma cells, developed by Beer et al..15] These strategies 2 employ microfluidic-based devices, which show promise to be 3 used for personalized pharmacological testing. Microfluidic 4 systems can be employed for the fabrication of drug delivery 5 systems having precisely controlled size and shape, rigidity as 6 well as drug-loading. In addition, microfluidic systems can be 7 efficiently used for the evaluation of drug-releasing prepara- 8 tions. ${ }^{[88]}$ Because organ-on-a-chip platforms can recapitulate 9 human physiology and pathophysiology, they can be effectively 10 helpful in translating new therapeutics to the clinic including 11 advanced nanomedicine. ${ }^{[19]}$

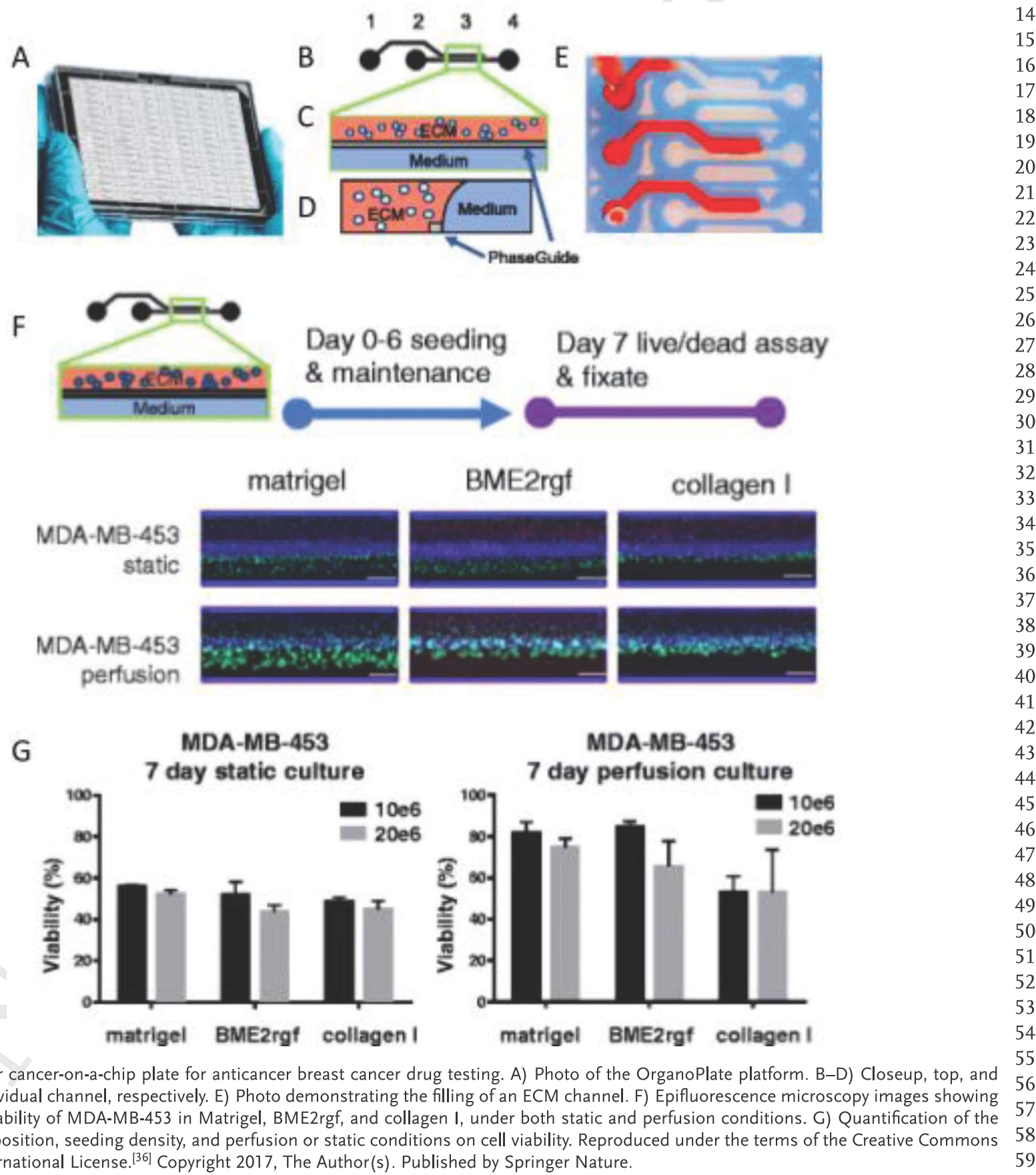


Table 1. Summary of cancer-on-chip studies.

\begin{tabular}{|c|c|c|}
\hline No. & Cancer type & Method \\
\hline 1. & Breast cancer & $\begin{array}{l}\text { Microfluidic 3D in vitro model for breast cancer metas- } \\
\text { tasis to bone }\end{array}$ \\
\hline 2. & Breast cancer & $\begin{array}{l}\text { 3D bone-on-chip for bone metastasis study of breast } \\
\text { cancer cells }\end{array}$ \\
\hline 3. & Breast cancer & $\begin{array}{l}\text { Disease-on-a-chip model in which cancer grows within } \\
\text { phenotypically normal breast luminal epithelium on } \\
\text { semicircular acrylic supports }\end{array}$ \\
\hline 4. & Breast cancer & $\begin{array}{c}\text { 3D high-throughput microfluidic platform for screening } \\
\text { of three triple negative breast cancer lines against } \\
\text { several anticancer drugs }\end{array}$ \\
\hline 5. & $\begin{array}{l}\text { Cancer immune } \\
\text { interactions }\end{array}$ & $\begin{array}{l}\text { Organ-on-chip tool to evaluate cancer-immune cell } \\
\text { interactions }\end{array}$ \\
\hline 6. & Lung cancer & $\begin{array}{l}\text { Multiorgan microfluidic chip mimicking in vivo micro- } \\
\text { environment of lung cancer metastasis }\end{array}$ \\
\hline 7. & Lung & $\begin{array}{l}\text { Organ-on-chip model recapitulates orthotopic lung } \\
\text { cancer growth and therapeutic response. }\end{array}$ \\
\hline 8. & $\begin{array}{l}\text { Pancreatic ductal } \\
\text { adeno-carcinoma }\end{array}$ & $\begin{array}{l}\text { Microfluidic 3D platform for culturing pancreatic ductal } \\
\text { adenocarcinoma cells }\end{array}$ \\
\hline
\end{tabular}
adenocarcinoma cells

Hassell et al. find that microenvironmental cues elicited by cells, as well as mechanical cues, significantly influence nonsmall-cell lung cancer growth in vitro. ${ }^{[60]}$ More importantly, their data demonstrate the ability of orthotopic cancer chip models to mimic growth patterns observed in vivo in patients and is consistent with that of human clinical trials, a feature which had not previously been recapitulated in vitro. ${ }^{[6]}$ Other strategies to mimic the TME include the use of multicellular aggregates ("microtumors") of subtype-specific breast cancer cells, by Singh et al., ${ }^{[31]}$ human-on-chip microvascular assay for visualization of tumor cell extravasation dynamics, by Chen et al., ${ }^{[76]}$ as well as development of an array of gut-ona-chips for drug development. ${ }^{[89]}$ The ultimate value in these new developments lies in their potential to allow for highthroughput drug screening of chemotherapeutics on ex vivo models of individual patients' tumors.

\section{High-Throughput Cancer-on-Chip Studies for Large Data Generation}

Cancer-on-a-chip models are advantageous for preclinical drug screening, as they can be designed to allow for high-throughput analysis of antitumor drug response and other biological parameters. ${ }^{[19,90]}$ In order to generate the large quantities of data needed to appropriately predict drug efficacy and potential side effects, as seen in clinical trials, high-throughput systems need to be developed. With the use of cancer-on-a-chip platforms, this type of large data creation and large-scale analysis is possible.

\subsection{Large Data Generating Cancer-on-Chip Studies}

In order to perform high-throughput studies, a large number of devices must be fabricated with high fidelity, reproducibility,

\section{Result \\ $3 D$ in vitro data on extravasation and micrometas- \\ tasis generation of breast cancer cells within bone microenvironment \\ Unique hallmarks of breast cancer bone in colonization Hao et al. 2018 [50] observed, previously only seen in vivo}

Mimicry of tumor environment provides a framework Vidi et al. 2014 [54] for the design and test of anticancer therapies.

High-throughput organ on a chip platform to select Lanz et al. 2017 [12] therapies in personalized medicine

Lanzet at.

Quantitative confirmation of the essential role of FPR1 Biselli et al. $2017 \quad[60]$ in cancer chemotherapy response

Multiorgan system provides useful tool to investigate Xu et al. $2016 \quad$ [53] cell-cell interactions in metastasis.

Discovery of mechanical stimuli dependent TKI therapy Hassell 2017 [52] 20

$$
\text { resistance }
$$

et al.

$2017+13]$

Growth characteristics closer to those of cells grown as Beer et al. $2017 \quad$ [13] spheroids than as classical $2 \mathrm{D}$ in vitro cultures

and homogeneity or a device must have the capacity to run 25 many tests on a single chip. In 2017, Chen et al. published a 26 protocol extension describing the fabrication of a microfluidic 27 device for modeling early metastasis in Nature Protocols. ${ }^{[76]} 28$ Their device was made from PDMS and featured three 29 hydrogel regions, separated by channels for media. Microposts 30 marked the device between each region. The central region 31 was filled with a fibrin gel and HUVEC suspension and the 32 two peripheral regions were filled with a fibrin gel and human 33 lung fibroblast suspension. Their device served as a model for 34 microcirculation, representing transendothelial migration and 35 early metastasis. They reported the capacity to fabricate and 36 seed up to 36 devices at a time without impacting cell viability. 37 Coupled with rapid quantification, their large number of 38 devices per experiment is expected to allow for high parametric 39 and throughput study, generating a large quantity of data. ${ }^{[86]} \quad 40$

To address the challenges associated with current in vitro 41 and in vivo preclinical models, such as the need for large 42 numbers of cell or animal materials, researchers have devel- 43 oped microdevices with up to thousands of microwells, 44 allowing for high-throughput testing. In 2014, Zhang et al. 45 developed a microfluidic device with 4000 ultraminiaturized 46 wells for high-throughput monitoring of chemotactic migra- 47 tion and invasion. ${ }^{[1]}$ Their multiwell invasion (MI) chip was 48 fabricated from PDMS using photolithography. The MI chip 49 was comprised of four compartments, each containing 10, 50 $10 \times 10$ microwell arrays, equaling 4000 microwells. They fabri- 51 cated both round $(200 \mu \mathrm{m}$ diameter $)$ and square $(200 \times 200 \mu \mathrm{m}) \quad 52$ wells, with a depth of $160 \mu \mathrm{m}$. The MI chips were used to per- 53 form 3D cell invasion assays with breast cancer cell lines, to 54 validate the chip's capacity to be used as a model for studying 55 metastatic breast cancer. The MI chip used a small cell sample 56 size (less than 1000 cells), allowing it to be used in the future 57 with limited cell sources, such as primary tumor cell samples. 58 The chip can be used in the future to run many tests at one 59 


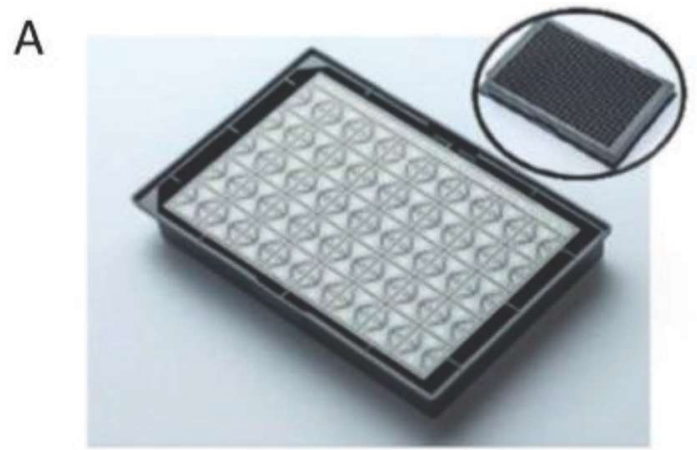

B
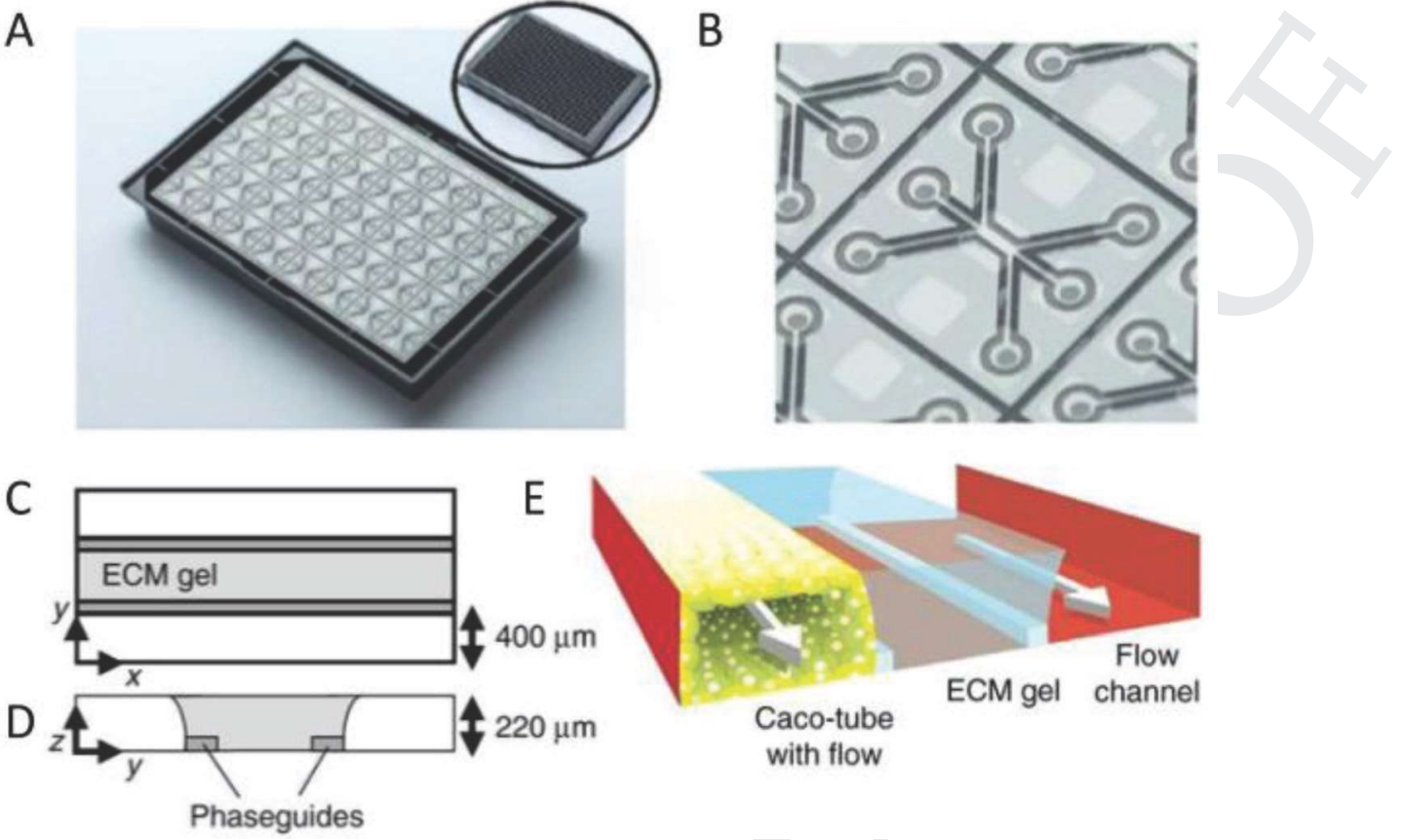

Figure 5. A) Photograph of the bottom of an OrganoPlate, showing 40 microfluidic channel networks and the top of a 384 well plate device. B) Zoomed in photograph of a single microfluidic channel network, with three channels joining in the center. C,D) Horizontal and vertical cross section. E) 3D sketch of the chip, comprised of a tubule, an extracellular matrix gel, and a perfusion lane. Reproduced under the terms of the Creative Commons Attribution 4.0 International License. ${ }^{[89]}$ Copyright 2017, The Author(s). Published by Springer Nature.

time or run tests on rare samples, making it a useful future tool for clinicians to evaluate the behavior of cancer cells and anticancer drug regimens.

Another method for generating large data sets via organ-ona-chip is the fabrication of multiple models on a single device. To our knowledge, there is not currently published literature joining multiple cancer-on-a-chip platforms. However, groups have multiplexed organ-on-a-chip devices together for other applications. Work in the organ-on-a-chip field reports the use of an array of gut-on-a-chip devices joined together, comprising a total of 357 gut tubes. ${ }^{[89]}$ The OrganoPlate (shown in Figure 5) is a 384-well plate platform housing 40 networks of microfluidic channels. Each OrganoPlate housed 40 epithelial gut tubes, which were tested against drug compounds at different concentrations to study the effect on epithelial barrier integrity. The study generated over 20000 data points, making it the largest reported organ-on-chip data set thus far. This study's highthroughput nature shows the promise of organ-on-a-chip platforms for use as new, efficient, and reliable preclinical models, with applications in anticancer drug testing. ${ }^{[63,89,92]}$

\subsection{Large Data Management and Extraction of Information}

With high-throughput platforms, such as organ-on-a-chip, generating unprecedented quantities of data, there lies the need for appropriate data management and analysis systems. While cancer-on-a-chip platforms have been rapidly developing, machine learning algorithms to manage these data have been developing in parallel. New data management strategies should incorporate four core pillars. The first pillar in data manage- ment is the hardware implementation of microchips, with 29 appropriate sensors and microsystems to measure the desired 30 parameters. The second pillar of large data management is data 31 collections, transmission, and storage. The third pillar required 32 is advanced machine learning algorithms to extract information 33 from the available huge data sets. Finally, the fourth pillar con- 34 cerns the interpretation of obtained data and its applications in 35 the discovery of new theories.

Cancer-on-a-chip platform sensors continuously measure 37 attributes of all cells within the device. The data flow can be of 38 extremely high volume; for example, if there are 50000 cells 39 under investigation, each with 20 measured factors $\mathrm{min}^{-1}$, then 40 one would receive $\approx 2 \mathrm{~GB}$ of data each day (variable by the coding 41 method of the collected data). With several parallel platforms 42 and prolonged data collection (potentially months of collection), 43 the size of the collected data may be exceedingly large. 44

After data collection, the next large stage is the big data 45 processing. For this purpose, we rely on highly sophisticated 46 ML algorithms, which can be divided into two categories: 47 supervised or unsupervised learning. ${ }^{[93]}$ These algorithms take 48 into consideration the number of cells $(N)$, number of param- 49 eters or attributes $(M)$, and the number of samples $(T)$. In this 50 example, we can define health status as $\mathrm{H}$ and assign binary 51 values of 0 (dead cell) and 1 (fully healthy cell). After collecting 52 big size data, we can build some model (dynamic or otherwise) 53 for the relationship between cellular attributes and health. The 54 data are typically divided into two parts, with one part (the 55 learning portion) used for modeling and the second used for 56 validation; the first part should be large enough in size to cap- 57 ture important mapping relations between the attributes and 58 the output status H. Other alternative ML algorithms to be used 59 
include recurrent type networks with deep learning structure. There are many ML algorithms with different concepts and criteria. A crucial component to any model, however, is keeping the complexity at enough to obtain a good generalized model but avoid creating unnecessary complexity, which may give high errors in the validation and testing phase.

There are other alternative approaches to supervised learning which are not discussed in this review. More information regarding ML algorithms can be found in other sources. ${ }^{\text {[93] }}$ Another challenge of big data processing is the interpretation of the ML algorithm results. Advanced artificial intelligence (AI) algorithms may be used to help human experts interpret ML outcomes. The discussed methodologies will aid in the processing and interpretation of data collected from future highthroughput, large data generating studies. With real-time data collection and processing, in the future, researchers will be able to perform trials on millions of therapeutic agents against a patient's specific tumor. Microfluidics, sensors, computing facilities, smart algorithms, and intelligent microautomated systems can be joined as the basis of advanced systems for next-generation anticancer drug design and development. ${ }^{[57,94]}$

\section{Challenges and Future Work}

Recent studies involving cancer-on-a-chip technologies have made great strides in better recapitulating the TME and in vivo cancer microenvironment. However, there is still work to be done identifying the fundamental TME elements needed to better mimic cancer tissue, for both the study of cancer biology and improved predictive ability of preclinical models for anticancer drug development. The development of personalized cancer-on-a-chip platforms for patients' primary tumor tissues will be a large step forward in harnessing the capabilities of cancer-on-a-chip platforms. This advancement will result in precision medicine and personalized oncology. Incorporation of TME elements, such as oxygen concentration and cytokine concentration gradients, will increase the complexity of canceron-a-chip models, improving the predictive power of these platforms. Once developed, there will likely be many challenges in the adoption of these technologies, that is, standardization and validation against current models.

With the adoption of organ-on-a-chip technologies, future oncology treatment is expected to be vastly different than today's regimens. As we move toward personalized oncology models, one predicted outcome is the use of patient-derived cells and extracted ECMs in chip technologies, capturing the biochemical, biophysical, and mechanical cues of the in vivo human cancer microenvironment. Another advantage of cancer-on-a-chip technologies is the capability for high-throughput, personalized screening of anticancer drug treatment and therapies. The capabilities of these platforms may also be expanded and used for more innovative cancer detection, ${ }^{[51]}$ for example, on-chip blood tests to replace bone biopsies for multiple myeloma. ${ }^{[81]}$

\section{Conclusions}

Once cancer-on-a-chip technologies are fully realized, the current regimented practice of choosing specific chemotherapeutics based solely on tumor type will seem impre- 1 cise and inaccurate. Personalized cancer chemotherapy will 2 eventually be adopted and the use of cancer-on-a-chip models 3 will become the clinical standard, allowing for more precise 4 and individual function-based selection of chemotherapeutics. 5 After the predictive power of these models are demonstrated, 6 on-a-chip tests will serve a central role in the development and 7 approval of new cancer therapeutics, replacing current pre- 8 clinical models. Adoption of these new technologies will both accelerate and decrease the costs of the drug development process and increase the precision of cancer therapy, benefiting patients, physicians, and care providers, as well as pharmaceutical and insurance companies.

\section{Acknowledgements}

The authors acknowledge funding from the National Institutes of Health (AR057837, AR066193, EB021857-01A1, HL137193-01, EB024403-01-01, CA214411-01A1, EB023052-01A1, HL140618-01, GM126831, HL140951, AR073135, and AR069564), Department of Defense: BiofabUSA Quick Start Project, and American Heart Association Transformational Project Award (18TPA34230036)

\section{Conflict of Interest}

The authors declare no conflict of interest.

\section{Keywords}

artificial intelligence, cancer models, chemotherapy, microfluidics, organ-on-a-chip

Received: April 20, 2019

Revised: July 22, 2019 Published online:

[1] Global Cancer Facts \& Figures, 4th ed., American Cancer Society, Atlanta, GA 2018.

[2] R. L. Siegel, K. D. Miller, A. Jemal, Ca-Cancer J. Clin. 2018, 68, 7.

[3] $\mathrm{NCl}$ Dictionary of Cancer Terms, National Institute of Health, Bethesda, MD

[4] Y.-H. V. Ma, K. Middleton, L. You, Y. Sun, Microsyst. Nanoeng. 2018, 4, 17104.

[5] P. S. Steeg, Nat. Rev. Cancer 2016, 16, 201

[6] D. S. Shewach, R. D. Kuchta, Chem. Rev. 2009, 109, 2859.

[7] S. J. Hachey, C. C. W. Hughes, Lab Chip 2018, 18, 2893.

[8] K. Smietana, M. Siatkowski, M. Moller, Nat. Rev. Drug Discovery 2016, 15, 379 .

[9] I. Kola, J. Landis, Nat. Rev. Drug Discovery 2004, 3, 711.

[10] T. L. Whiteside, Oncogene 2008, 27, 5904.

[11] M. Wang, J. Zhao, L. Zhang, F. Wei, Y. Lian, Y. Wu, Z. Gong, S. Zhang, J. Zhou, K. Cao, X. Li, W. Xiong, G. Li, Z. Zeng, C. Guo, J. Cancer 2017, 8, 761.

[12] M. A. Swartz, N. lida, E. W. Roberts, S. Sangaletti, M. H. Wong, F. E. Yull, L. M. Coussens, Y. A. DeClerck, Cancer Res. 2012, 72, 2473.

[13] Z. Wang, R. Samanipour, K. Kim, in Biomedical Engineering: Frontier Research and Converging Technologies (Eds: H. Jo, H.-W. Jun, J. Shin, 3

.
8 
S. Lee), Springer International Publishing, Cham, Switzerland 2016 , pp. 209-233

[14] M. Kapałczyńska, T. Kolenda, W. Przybyła, M. Zajączkowska, A. Teresiak, V. Filas, M. Ibbs, R. Bliźniak, Ł. Łuczewski, K. Lamperska, Arch. Med. Sci. 2018, 14, 910.

[15] M. Beer, N. Kuppalu, M. Stefanini, H. Becker, I. Schulz, S. Manoli, J. Schuette, C. Schmees, A. Casazza, M. Stelzle, A. Arcangeli, Sci. Rep. 2017, 7, 1325.

[16] R. Chakrabarti, Y. Kang, Methods Mol. Biol. 2015, 1267, 367.

[17] M. Cekanova, K. Rathore, Drug Des., Dev. Ther. 2014, 8, 1911.

[18] C.-P. Day, G. Merlino, T. Van Dyke, Cell 2015, 163, 39

[19] Y. S. Zhang, Y. N. Zhang, W. Zhang, Drug Discovery Today 2017, 22, 1392.

[20] Z. Xu, E. Li, Z. Guo, R. Yu, H. Hao, Y. Xu, Z. Sun, X. Li, J. Lyu, Q. Wang, ACS Appl. Mater. Interfaces 2016, 8, 25840.

[21] R. Portillo-Lara, N. Annabi, Lab Chip 2016, 16, 4063.

[22] G. Urban, K. M. Bache, D. Phan, A. Sobrino, A. K. Shmakov, S. J. Hachey, C. Hughes, P. Baldi, IEEE/ACM Trans. Comput. Biol. Bioinf. 2018, 16, 1029.

[23] M. Dhandapani, A. Goldman, J. Mol. Biomark. Diagn. 2017, 8, 356

[24] J. W. Gray, G. B. Mills, Cancer Discovery 2015, 5, 1130.

[25] S. Breslin, L. O'Driscoll, Drug Discovery Today 2013, 18, 240.

[26] K. Duval, H. Grover, L.-H. Han, Y. Mou, A. F. Pegoraro, J. Fredberg, Z. Chen, Physiology 2017, 32, 266

[27] F. Pampaloni, E. G. Reynaud, E. H. Stelzer, Nat. Rev. Mol. Cell Biol. 2007, 8, 839 .

[28] M. E. Katt, A. L. Placone, A. D. Wong, Z. S. Xu, P. C. Searson, Front. Bioeng. Biotechnol. 2016, 4, 12

[29] H.-F. Tsai, A. Trubelja,A. Q. Shen, G. Bao, 2017, 14, 20170137.

[30] C. R. Justus, N. Leffler, M. Ruiz-Echevarria, L. V. Yang, J. Visualized Exp. 2014, 51046

[31] M. Singh, S. Mukundan, M. Jaramillo, S. Oesterreich, S. Sant, Cancer Res. 2016, 76, 3732

[32] B. A. Ruggeri, F. Camp, S. Miknyoczki, Biochem. Pharmacol. 2014, $87,150$.

[33] Y. Lai, X. Wei, S. Lin, L. Qin, L. Cheng, P. Li, J. Hematol. Oncol. 2017, 10, 106

[34] M. R. Carvalho, D. Lima, R. L. Reis, V. M. Correlo, J. M. Oliveira, Trends Biotechnol. 2015, 33, 667.

[35] M. Moro, G. Bertolini, R. Caserini, C. Borzi, M. Boeri, A. Fabbri, G. Leone, P. Gasparini, C. Galeone, G. Pelosi, L. Roz, G. Sozzi, U. Pastorino, Sci. Rep. 2017, 7, 6689

[36] H. L. Lanz, A. Saleh, B. Kramer, J. Cairns, C. P. Ng, J. Yu, S. J. Trietsch, T. Hankemeier, J. Joore, P. Vulto, R. Weinshilboum, L. Wang, BMC Cancer 2017, 17, 709

[37] L. C. Kimlin, G. Casagrande, V. M. Virador, Mol. Carcinog. 2013, 52, 167.

[38] K. Ronaldson-Bouchard, G. Vunjak-Novakovic, Cell Stem Cell 2018, 22, 310 .

[39] G. M. Whitesides, Nature 2006, 442, 368

[40] A. K. Achyuta, A. J. Conway, R. B. Crouse, E. C. Bannister, R. N. Lee, C. P. Katnik, A. A. Behensky, J. Cuevas, S. S. Sundaram, Lab Chip 2013, 13, 542.

[41] J. A. Brown, V. Pensabene, D. A. Markov, V. Allwardt, M. D. Neely, M. Shi, C. M. Britt, O. S. Hoilett, Q. Yang, B. M. Brewer, P. C. Samson, L. J. McCawley, J. M. May, D. J. Webb, D. Li, A. B. Bowman, R. S. Reiserer, J. P. Wikswo, Biomicrofluidics 2015, 9, 054124.

[42] S. Musah, A. Mammoto, T. C. Ferrante, S. S. F. Jeanty, M. Hirano-Kobayashi, T. Mammoto, K. Roberts, S. Chung, R. Novak, M. Ingram, T. Fatanat-Didar, S. Koshy, J. C. Weaver, G. M. Church, D. E. Ingber, Nat. Biomed. Eng. 2017, 1, 0069.

[43] K. J. Jang, A. P. Mehr, G. A. Hamilton, L. A. McPartlin, S. Chung, K. Y. Suh, D. E. Ingber, Integr. Biol. 2013, 5, 1119

[44] H. J. Kim, D. E. Ingber, Integr. Biol. 2013, 5, 1130.
[45] H. J. Kim, D. Huh, G. Hamilton, D. E. Ingber, Lab Chip 2012, 12, 1 2165.

[46] D. Huh, B. D. Matthews, A. Mammoto, M. Montoya-Zavala, 3 H. Y. Hsin, D. E. Ingber, Science 2010, 328, 1662.

[47] R. Booth, H. Kim, Lab Chip 2012, 12, 1784.

[48] F. Zheng, F. Fu, Y. Cheng, C. Wang, Y. Zhao, Z. Gu, Small 2016, 12, 5 2253.

[49] Y. S. Zhang, F. Davoudi, P. Walch, A. Manbachi, X. Luo, V. Dell'Erba, 7 A. K. Miri, H. Albadawi, A. Arneri, X. Li, X. Wang, M. R. Dokmeci, 8 A. Khademhosseini, R. Oklu, Lab Chip 2016, 16, 4097.

[50] A. Jain, R. Barrile, A. D. van der Meer, A. Mammoto, T. Mammoto, 10 K. De Ceunynck, O. Aisiku, M. A. Otieno, C. S. Louden, 11 G. A. Hamilton, R. Flaumenhaft, D. E. Ingber, Clin. Pharmacol. Ther. 12 2018, 103, 332.

[51] G. Kaushik, J. Leijten, A. Khademhosseini, Stem Cells 2017, 35, 51.

[52] B. Zhang, A. Korolj, B. F. L. Lai, M. Radisic, Nat. Rev. Mater. 2018, 15 3,257 .

[53] K. Fetah, P. Tebon, M. J. Goudie, J. Eichenbaum, L. Ren, 16 N. Barros, R. Nasiri, S. Ahadian, N. Ashammakhi, M. R. Dokmeci, 17 A. Khademhosseini, Prog. Biomed. Eng. 2019, 1, 012001.

[54] T. Takebe, B. Zhang, M. Radisic, Cell Stem Cell 2017, 21, 297.

[55] J. J. F. Sleeboom, H. E. Amirabadi, P. Nair, C. M. Sahlgren, 20 J. M. J. den Toonder, Dis. Models Mech. 2018, 11, dmm033100. 21

[56] A. Sontheimer-Phelps, B. A. Hassell, D. E. Ingber, Nat. Rev. Cancer 22 2019, 19, 65 .

[57] M. Elmusrati, N. Ashammakhi, J. Craniofac. Surg. 2018, 29, 1682.

[58] S. Hao, L. Ha, G. Cheng, Y. Wan, Y. Xia, D. M. Sosnoski, 25 A. M. Mastro, S. Y. Zheng, Small 2018, 14, 1702787.

[59] S. Bersini, J. S. Jeon, G. Dubini, C. Arrigoni, S. Chung, J. L. Charest 26 M. Moretti, R. D. Kamm, Biomaterials 2014, 35, 2454, 27

[60] B. A. Hassell, G. Goyal, E. Lee, A. Sontheimer-Phelps, O. Levy, 28 C. S. Chen, D. E. Ingber, Cell Rep. 2017, 21, 508 . 29

[61] P. A. Vidi, T. Maleki, M. Ochoa, L. Wang, S. M. Clark, J. F. Leary, 30 S. A. Lelievre, Lab Chip 2014, 14, 172.

[62] B. Emon, J. Bauer, Y. Jain, B. Jung, T. Saif, Comput. Struct. 32 Biotechnol. J. 2018, 16, 279.

[63] J. Ahn, Y. J. Sei, N. L. Jeon, Y. Kim, Bioengineering 2017, 4, 64.

[64] P. Parente, P. Parcesepe, C. Covelli, N. Olivieri, A. Remo, 34 M. Pancione, T. P. Latiano, P. Graziano, E. Maiello, G. Giordano, Gastroenterol. Res. Pract. 2018, 2018, 7530619.

[65] S. Upadhyay, N. Sharma, K. B. Gupta, M. Dhiman, J. Cell. Biochem. 37 2018, 119, 5028

[66] E. Biselli, E. Agliari, A. Barra, F. R. Bertani, A. Gerardino, A. De 39 Ninno, A. Mencattini, D. Di Giuseppe, F. Mattei, G. Schiavoni, 40 V. Lucarini, E. Vacchelli, G. Kroemer, C. Di Natale, E. Martinelli, 41 L. Businaro, Sci. Rep. 2017, 7, 12737.

[67] E. Agliari, E. Biselli, A. De Ninno, G. Schiavoni, L. Gabriele, 43 A. Gerardino, F. Mattei, A. Barra, L. Businaro, Sci. Rep. 2015, 4, 44 6639.

[68] Y. Zheng, Y. Sun, X. Yu, Y. Shao, P. Zhang, G. Dai, J. Fu, Adv. Healthcare Mater. 2016, 5, 1014

[69] M. Pisano, V. Triacca, K. A. Barbee, M. A. Swartz, Integr. Biol. 2015, 7, 525

[70] K. Mittal, J. Ebos, B. Rini, Semin. Oncol. 2014, 41, 235.

[71] C. L. Chaffer, R. A. Weinberg, Science 2011, 331, 1559

[72] C Yates, C R Shepard, C. Papworth, A. Dash, D. B. Stolz, S. Tannenbaum, L. Griffith, A. Wells, Adv. Cancer Res. 2007, 97, 52 225.

[73] S. Kim, M. Chung, N. L. Jeon, Biomaterials 2016, 78, 115.

[74] H. Lee, W. Park, H. Ryu, N. L. Jeon, Biomicrofluidics 2014, 8, 054102.

[75] I. K. Zervantonakis, S. K. Hughes-Alford, J. L. Charest, J. S. Condeelis, F. B. Gertler, R. D. Kamm, Proc. Natl. Acad. Sci. USA 2012, 109, 13515.

[76] M. B. Chen, J. A. Whisler, J. Frose, C. Yu, Y. Shin, R. D. Kamm, Nat. 58 Protoc. 2017, 12, 865 
[77] J. S. Jeon, S. Bersini, M. Gilardi, G. Dubini, J. L. Charest, M. Moretti, R. D. Kamm, Proc. Natl. Acad. Sci. USA. 2015, 112, 214.

[78] Q. Zhang, T. Liu, J. Qin, Lab Chip 2012, 12, 2837.

[79] J. S. Jeon, I. K. Zervantonakis, S. Chung, R. D. Kamm, J. L. Charest, PLoS One 2013, 8, e56910.

[80] K. F. Lei, H. P. Tseng, C. Y. Lee, N. M. Tsang, Sci. Rep. 2016, 6, 25557.

[81] Y.-C. Toh, A. Raja, H. Yu, D. van Noort, Bioengineering 2018, 5, 29.

[82] D. Caballero, S. Kaushik, V. M. Correlo, J. M. Oliveira, R. L. Reis, S. C. Kundu, Biomaterials 2017, 149, 98.

[83] E. T. Roussos, J. S. Condeelis, A. Patsialou, Nat. Rev. Cancer 2011, 11, 573.

[84] A. Aung, J. Theprungsirikul, H. L. Lim, S. Varghese, Lab Chip 2016, 16,1886

[85] R. Huang, W. Zheng, W. Liu, W. Zhang, Y. Long, X. Jiang, Sci. Rep. 2015, 5, 17768.
[86] S. Sart, R. F. X. Tomasi, G. Amselem, C. N. Baroud, Nat. Commun. 1 2017, 8, 469 .

[87] Y. Choi, E. Hyun, J. Seo, C. Blundell, H. C. Kim, E. Lee, S. H. Lee, 3 A. Moon, W. K. Moon, D. Huh, Lab Chip 2015, 15, 3350.

[88] H. Zhang, Y. Zhu, Y. Shen, Small 2018, 14, 1800360.

[89] S. J. Trietsch, E. Naumovska, D. Kurek, M. C. Setyawati, M. K. Vormann, K. J. Wilschut, H. L. Lanz, A. Nicolas, C. P. Ng, J. Joore, S. Kustermann, A. Roth, T. Hankemeier, A. Moisan, P. Vulto, Nat. Commun. 2017, 8, 262.

[90] N. Kashaninejad, M. R. Nikmaneshi, H. Moghadas, A. K. Oskouei, 9 M. Rismanian, M. Barisam, M. S. Saidi, B. Firoozabadi, 10 Micromachines 2016, 7, 130.

[91] Y. Zhang, L. Zhou, L. Qin, J. Am. Chem. Soc. 2014, 136, 15257.

[92] N. Ashammakhi, M. Elmusrati, bioXriv 2018, 273847.

[93] E. Alpaydin, Introduction to Machine Learning, The MIT Press, Cambridge, MA 2016.

[94] E. Kongadzem, Parallel Faculty of Technology and Innovations, University of Vassa, Vassa, Finland 2018. 